\title{
El impacto económico-territorial del turismo internacional por vía aérea (2010-2017)
}

\author{
GRISEL VICTORIA AZCUY
}

GEOTRAER, Instituto de Geografía, Universidad de Buenos Aires, Argentina.

griselazcuy@gmail.com

\section{RESUMEN}

Cuando se estudia la relación entre el transporte aéreo y la actividad turística suele ponerse el foco en los impactos económicos positivos generados por la atracción de turismo receptivo. Sin embargo, así como los servicios aéreos pueden incentivar la llegada de visitantes, también facilitan la salida de turistas residentes hacia el exterior. El presente trabajo examina la evolución reciente de la red aerocomercial internacional argentina y los cambios en el marco regulatorio -tendientes a lograr una mayor apertura y liberalización del mercado- en relación con el impacto económico generado por el turismo aéreo internacional. A través del uso de estadísticas aerocomerciales y encuestas sobre turismo se identificó un incremento sostenido del impacto negativo del turismo por vía aérea reforzado por la nueva configuración de la red de servicios. A este déficit de la balanza comercial turística por vía aérea se sumó un desequilibrio en la cuenta corriente de servicios aéreos relacionado con la remisión de utilidades por parte de las compañías extranjeras hacia el exterior.

PALABRAS CLAVE: transporte aéreo; turismo; impacto económico-territorial; liberalización; balanza comercial turística.

Recepción: 21/02/2018 | Evaluación: 02/09/2018 | Aceptación: 28/10/2018 


\section{ABSTRACT}

THE ECONOMIC-TERRITORIAL IMPACT OF INTERNATIONAL TOURISM BY AIRWAY (2010-2017)

When studying the relationship between air transport and tourist activity, the focus is usually on the positive economic impacts generated by the attraction of receptive tourism. However, just as air services can encourage the arrival of visitors, they also facilitate the departure of resident tourists abroad. This paper examines the recent evolution of the Argentine international airport network and the changes in the regulatory framework - aimed at achieving greater market opening and liberalization - in relation to the economic impact generated by international air tourism. Through the use of aero-commercial statistics and tourism surveys, a sustained increase in the negative impact of tourism by air was reinforced, reinforced by the new configuration of the service network. To this deficit of the tourist trade balance by air was added an imbalance in the current account of air services related to the remittance of profits by foreign companies abroad.

KEYWORDS: air transport; tourism; economic-territorial impact; liberalization; tourist trade balance. 


\section{RESUMO}

\section{O IMPACTO ECONÔMICO-TERRITORIAL DO TURISMO INTERNACIONAL POR VIA AÉREA (2010-2017)}

Ao ESTUDAR a relação entre transporte aéreo e atividade turística, o foco geralmente está nos impactos econômicos positivos gerados pela atração do turismo receptivo. No entanto, assim como os serviços aéreos podem incentivar a chegada de visitantes, eles também facilitam a saída de turistas residentes no exterior. Este artigo examina a recente evoluçâo da rede comercial aérea internacional da Argentina e as mudanças no marco regulatório - visando maior abertura e liberalização do mercado - em relaçáo ao impacto econômico gerado pelo turismo aéreo internacional. Com o uso de estatísticas aero-comerciais e pesquisas de turismo, foi reforçado um aumento sustentado do impacto negativo do turismo aéreo, reforçado pela nova configuraçáo da rede de serviços. A esse déficit da balança comercial de turistas por via aérea foi adicionado um desequilíbrio na conta corrente de serviços aéreos relacionada à remessa de lucros por empresas estrangeiras ao exterior.

PALAVRAS-CHAVES: transporte aéreo; turismo; impacto econômico-territorial; liberalização; balança comercial turística. 


\section{Introducción}

En los últimos años, el estudio del impacto económico generado por el transporte aéreo en el Producto Bruto Interno (PBI) mundial ha cobrado especial relevancia. En 2017, diversos organismos internacionales vinculados al sector aéreo publicaron por primera vez un documento unificado sobre los beneficios de la aviación a nivel mundial. Según este informe, el impacto económico total de la aviación en 2014 alcanzó los $\$ 2,7$ billones de dólares, alrededor del 3,5\% del producto bruto mundial. Dentro de este monto, se estimó que el turismo por vía aérea generó un impacto positivo de $\$ 892,4$ billones de dólares (Airports Council International Europe, ACI-Europe. ACI et al., 20I7:9). Ese mismo año, International Air Transport Association (IATA, 20I7) publicó un informe sobre los beneficios de la aviación en Argentina. Según sus estimaciones, en el año 2014 los turistas extranjeros que llegaron por vía aérea generaron una contribución de \$4.IOO millones de dólares al PBI argentino y sostuvieron más de 130 mil empleos. En el ámbito nacional, el Organismo Regulador del Sistema Nacional de Aeropuertos (ORSNA) también realizó una serie de estudios sobre el impacto económico-territorial de los principales aeropuertos argentinos contabilizando cuál fue el aporte económico total que generó cada terminal aeroportuaria durante el año 20ı3, en su área de influencia.

La metodología utilizada para la cuantificación de los efectos económicos posibilitados por la aviación define tradicionalmente impactos directos, indirectos, inducidos y catalíticos, teniendo en cuenta los ingresos/valor agregado, las ganancias, la recaudación impositiva y los empleos generados por el transporte aéreo y sus actividades afines (Lipovich, 20IO). Dentro de los efectos catalíticos se destaca la actividad turística como uno de los sectores económicos más potenciados por la aviación ya que el modo aéreo permite conectar distintas regiones del planeta en muy poco tiempo y a bajo costo relativo. Bajo esa lógica, mejorar los niveles de conectividad y accesibilidad aéreas de un territorio promueve efectos positivos por la posibilidad de acceder a nuevos mercados, recursos turísticos y ampliar las experiencias culturales y sociales.

Ahora bien, generalmente estos enfoques suelen expresar una dinámica causa-efecto entre la oferta de servicios aéreos y los beneficios económico-sociales. Especifícame, vale la pena poner en discusión algunos supuestos involucrados en la relación transporte aéreo y turismo. Por 
un lado, se hace énfasis en los impactos positivos que el transporte aéreo genera en la economía como consecuencia del gasto del turismo receptivo en la economía local. Sin embargo, se omite que los flujos aéreos son bidireccionales y, por lo tanto, también se producen impactos negativos asociados al turismo que viaja al exterior (Graham, I995; Lipovich, 2010).

Por otro, desde las cámaras empresarias, organismos internacionales, esferas de gobierno e incluso ámbitos académicos se promueve la liberalización del mercado aerocomercial como una herramienta clave para la promoción turística (Forsyth, 2008; UNWTO, 20I2; Duval, 20I3; IATA, 20I7). Sin embargo, aunque los regímenes de regulación inciden en la configuración de los mercados aéreos nacionales y en las estrategias operacionales que adoptan las líneas aéreas, existe poca evidencia que constate una causalidad clara entre la liberalización y el crecimiento del negocio turístico (Dobruszkes, et al., 2016).

Teniendo en cuenta estas tensiones, el objetivo de este trabajo es analizar los cambios producidos en la red aerocomercial internacional argentina entre 2010 y 2017 y sus efectos sobre la balanza comercial turística. Se pondrá especial atención en los principales cambios en la oferta de servicios internacionales asociados a modificaciones del marco regulatorio argentino y se adoptará una perspectiva de saldo de impactos del turismo -receptivo y emisivo- para poder entender el fenómeno de manera integral (Lipovich, 20IO).

\section{Transporte aéreo y turismo}

Indudablemente, uno de los principales factores de generación y atracción de viajes aéreos es el turismo. La Organización Mundial del Turismo señaló que en 2016 el 55\% del turismo mundial receptivo viajó por avión, el $39 \%$ por carretera, el $4 \%$ por vías navegables y el $2 \%$ por ferrocarril (OMT, 2017). A su vez, el sector turístico también se ha convertido en uno de los principales actores del comercio mundial y representa una de las principales fuentes de ingresos de numerosos países en desarrollo ${ }^{\mathrm{r}}$.

La relación entre el transporte aéreo y la actividad turística tiene como vector fundamental la combinación entre la necesidad de movilidad de las personas y las múltiples conexiones nacionales e internacionales

1 Recuperado de: http://www2.unwto.org/es/content/por-que-el-turismo (02/08/2018) 
provistas por la aviación. Paulatinamente, la expansión de la red aerocomercial ha ido mejorando la comunicación entre los centros emisores y los centros receptores de la demanda turística, y en particular de aquellos que se encuentran a mayores distancias posibilitando un flujo más dinámico (Wallingre, 20ro). Sería difícil considerar actualmente que un lugar turísticamente atractivo no cuente con un aeropuerto. La provisión adecuada de transporte aéreo es prácticamente una condición previa para la promoción del turismo como medio de desarrollo (Graham, 1995; Lipovich, 2010). La oferta de servicios aéreos permite la llegada de turistas extranjeros -usualmente de mayor poder adquisitivoposibilitando la generación de ingresos en divisas y oportunidades de empleo en una amplia gama de actividades como hotelería, restaurantes, entretenimiento, recreación, alquiler de automóviles, etc.

Las empresas de servicios, cuya rentabilidad depende de la facilidad de acceso a su clientela y/o de las economías de escala que proporciona el volumen de pasajeros de un aeropuerto -hoteles, salas de congresos, áreas comerciales, etc.- son favorecidas por la conectividad y accesibilidad aéreas (Gámir y Ramos, 2002). Por ello, mejorar la oferta de servicios aéreos mediante el incremento de frecuencias a destinos preexistentes, la mejora de la calidad de esos enlaces y la posibilidad de acceder a nuevos mercados económicamente importantes, permite expandir los vínculos sociales y económicos de una región (ACI-Europe, 20I5).

Sin embargo, la oferta aérea también puede vehiculizar contrapartidas negativas ya que posibilita la salida del turismo emisivo, la emigración de mano de obra altamente calificada, el ingreso de importaciones, la fuga de divisas o el aumento de precios para la población local por la llegada de extranjeros (Graham, 1995). Como contrapartida, la ACI-Europe (20I5) plantea que si bien los servicios aéreos facilitan el turismo de salida y la consecuente reducción de la cantidad de dinero gastado dentro de la economía, igualmente garantiza cierto gasto en la economía doméstica vía aerolíneas con base en el país, agentes de viajes locales, transporte hacia/desde el aeropuerto, etc. por lo que la contribución neta del turismo sería positiva.

Como balance, examinar el impacto económico que genera el turismo por vía aérea requiere adoptar una perspectiva integral donde se cuantifiquen los efectos positivos generados por el turismo receptivo y los negativos vinculados el turismo emisivo. El saldo entre ambos flujos representaría la contribución neta real del turismo en el territorio (Lipo- 
vich, 20IO). Los informes del impacto económico-territorial publicados por el ORSNA en 2015 incorporan dicho enfoque. Por ejemplo, para el caso cordobés en 2013 se contabilizó un impacto positivo del turismo receptivo (interno e internacional) de $\$ 1.764$ millones de pesos, mientras que el impacto negativo del turismo emisivo (interno e internacional) fue de \$ I.670 millones de pesos (ORSNA, 2015). Por lo que el saldo turístico para el área de influencia del aeropuerto de Córdoba fue de $\$ 93,7$ millones de pesos.

Ahora bien, los movimientos de pasajeros a través de cualquier sistema de transporte aéreo internacional se rigen a partir de tratados internacionales que especifican la naturaleza y la medida en que tales servicios están permitidos: rutas, capacidad, frecuencia y designación comercial (Duval, 20I8). Por lo tanto, los Estados tienen un rol central al determinar las pautas bajo las cuales se configura la red de servicios aéreos de un territorio y, en consecuencia, influyen en los niveles de conectividad y accesibilidad aéreas que puede suministrar la aviación repercutiendo en los flujos aéreos que se establecen entre un territorio determinado y el resto del mundo.

Usualmente, se promueve como herramienta de estímulo al turismo, la implementación de marcos regulatorios liberalizados, dado que uno de los procesos resultantes de la ejecución de dichas políticas es el aumento del número de vuelos, asientos, asientos-kilómetro y rutas operadas en el mercado. Sin embargo, se ha demostrado que en paralelo también se producen procesos de concentración y centralización empresarial de la oferta aérea y una concentración geográfica de los vuelos, traducida en una red de servicios fuertemente jerarquizada y con altos grados de selectividad territorial (Graham, 1995; Goetz y Vowles, 2009; Dobruszkes, 20I4; Bowen, 20I4; Lipovich, 20I4).

Los procesos de liberalización, desregulación y privatización del mercado aerocomercial que se iniciaron con la firma de la Airline Deregulation Act (1978) en Estados Unidos, produjeron grandes cambios organizacionales en el sector que impactaron de lleno en las estrategias de las líneas aéreas para adecuarse a un entorno más competitivo. A diferencia de lo que se esperaba, las grandes compañías terminaron ejerciendo economías de escala, de alcance y de densidad de tráfico como estrategias frente a la competencia abierta (Goetz, 2002). La reorganización de las redes aerocomerciales tuvo como eje la generación de centros concentradores de vuelos (hubs) frente a las tradicionales operaciones punto a punto. El desarrollo de redes hub-and-spoke trajo como conse- 
cuencia un proceso de concentración geográfica de los vuelos en ciertas ciudades dando lugar a un nuevo tipo de competencia entre aeropuertos para ofrecer servicios centrales (hubs) (Redondi et. al., 20II).

En este contexto, la reconfiguración del mercado aerocomercial bajo el paradigma vigente requiere repensar las formas que vehiculizan el impacto genuino de los servicios aéreos en el territorio. Al respecto, Lipovich (20I0) plantea que la oferta aerocomercial promueve impactos económicos positivos especialmente cuando se trata de vuelos brindados por líneas aéreas con base en el país y se desarrollan estrategias operativas basadas en hubs locales. El efecto económico genuino se relaciona con la propia facturación de la empresa nacional que genera valor agregado a la economía local, pero también con la contratación de mano de obra para garantizar las actividades de gestión, administración, ventas, planificación de vuelos, marketing, incluso de mantenimiento de la flota en aeropuertos locales. Además, la operación en hub promueve un escenario de sobreconectividad aérea que excede el tráfico propio de la ciudad, analizada como producto de la proliferación de conexiones aéreas, y que lleva a mejorar las condiciones de vinculación de las ciudades, potenciando de sobremanera su fertilidad urbana (Arias y Lipovich, 2017:135). En contraste, las compañías internacionales generan estos impactos en sus países de origen y en sus bases operativas en el exterior.

Por lo tanto, a la hora de pensar la relación entre el transporte aéreo y el turismo, es necesario tener en cuenta el marco regulatorio, la distribución geográfica de las redes aéreas, las características de las oferta, los actores que están involucrados en la reconfiguración de ese mercado, el rol del Estado a la hora de promover, limitar o incluso de actuar directamente a través de compañías aéreas y de aeropuertos propios en la concreción de ciertas estrategias operativas, y la bidireccionalidad del flujo turístico (receptivo/emisivo) posibilitado por las conexiones aéreas.

\section{Cambios en la red aerocomercial internacional}

Durante los últimos 20 años, la demanda internacional de pasajeros aéreos en Argentina mostró un incremento del I29\%, sumando más de 8,I89 millones de pasajeros al mercado. Pese al declive producido por la crisis económica, política y social del 200I, el sector aerocomercial comenzó un nuevo período de crecimiento sostenido a partir del 2003. 
Entre 2010 y 2017 los pasajeros internacionales crecieron un $40 \%$, pasando de 10,409 millones (2010) a I4,530 millones (2017) (Figura I). En ese período, el crecimiento interanual de la demanda internacional fue relativamente homogéneo, salvo entre 2016 y 2017 cuando se incorporaron $\mathrm{I}, 803$ millones de pasajeros internacionales $(+\mathrm{I} 4 \%)$.

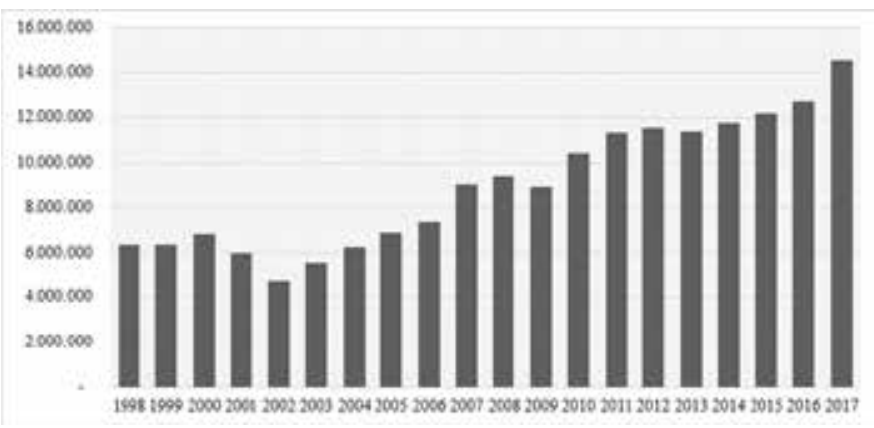

Fuente: INDEC.

Figura 1: Evolución de la demanda área de pasajeros internacionales en Argentina (1998-2017)

Desde el punto de vista de la oferta aérea, en el año 20 o la red aerocomercial internacional argentina contaba con 45 rutas: 36 salían o llegaban a Buenos Aires $(80 \%)$, mientras que $9(20 \%)$ conectaban directamente con Córdoba, Mendoza, Rosario y Salta. A fines del período (20I7), la cantidad de rutas ofrecidas desde/hacia el país había crecido un $39 \%$ llegando a 68 enlaces: 43 se operaban desde la Región Metropolitana de Buenos Aires- RMBA- (63\%) y 25 desde otros aeropuertos del país (37\%). Puntualmente, la incorporación de vuelos internacionales en ciudades por fuera de la RMBA fue un proceso que tomó vigor a partir de 20I6. Entre 20IO y 2015 la cantidad de enlaces hacia el exterior desde los aeropuertos provinciales -exceptuando Aeroparque y Ezeiza- se mantuvo relativamente estable. Sin embargo, entre 2015 y 2017 se sumaron 12 rutas nuevas. A partir de 2016, los cambios introducidos en el marco regulatorio del sector aerocomercial tuvieron fuerte incidencia en la reconfiguración territorial de los servicios aéreos. La nueva política aérea buscó ganar un mayor grado de liberalización y apertura del mercado aéreo a través de: la renegociación de acuerdos bilaterales, la habilitación de nuevas rutas y el aumento de frecuencias para compañías extranjeras, y la descentralización de la operación de los vuelos internacionales. Como resultado, 
las mayores transformaciones de la red internacional se produjeron a escala latinoamericana ${ }^{2}$.

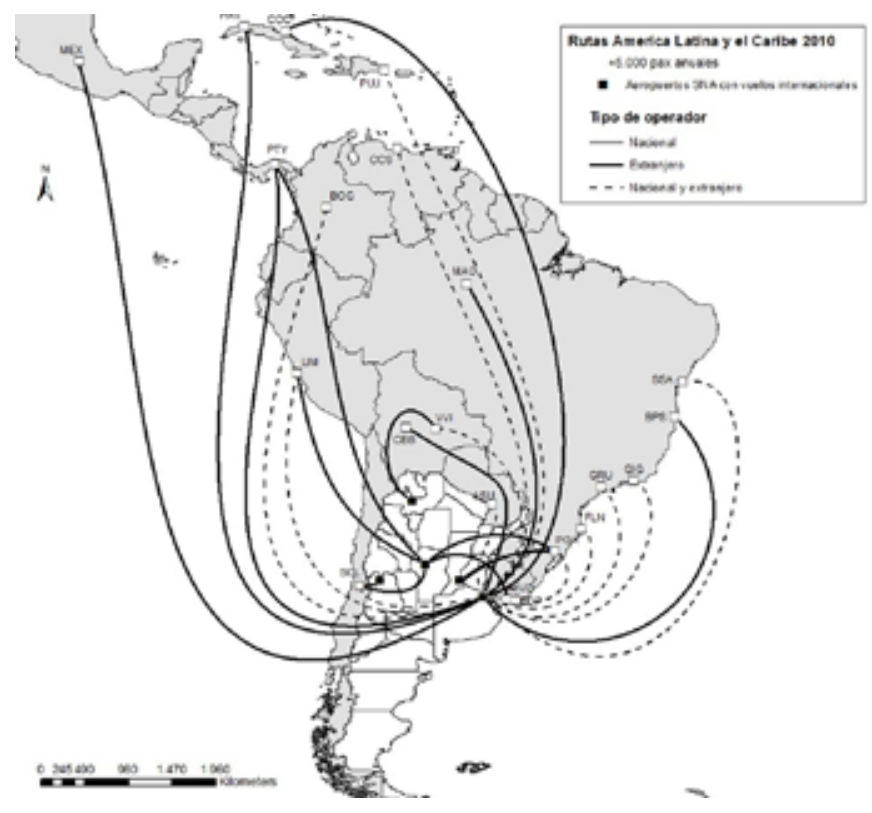

Figura 2. Red de vuelos internacionales desde/hacia América Latina y el Caribe (2010)
Fuente: Elaboración propia en base a datos de la Administración Nacional de Aviación Civil (ANAC)

Las Figuras 2, 3 y 4 permiten observar cómo fue mutando la morfología de los servicios aéreos de Argentina con América Latina y el Caribe a partir de momentos específicos: 20I0, 2015 y 20I7. Entre 20IO y 20I5, la cantidad de destinos operados se incrementó tanto en la RMBA como en el resto de los aeropuertos. Córdoba se mantuvo como la segunda ciudad más conectada con una red vinculada exclusivamente con los centros de operaciones de las principales empresas multilatinas: Santiago de Chile (LATAM), Lima (LATAM), Panamá (Copa Airlines), Sao Paulo (LATAM/Gol)

2 En materia de rutas extra-regionales las principales modificaciones tuvieron que ver con la pérdida de enlaces directos con Washington (América del Norte), Ciudad del Cabo, Johanesburgo (África) y Sydney (Oceanía) desde Buenos Aires y Madrid, desde Córdoba. Se sumaron Newark, Amsterdam y Londres. 
Fuente: Elaboración propia en base a datos de la ANAC.

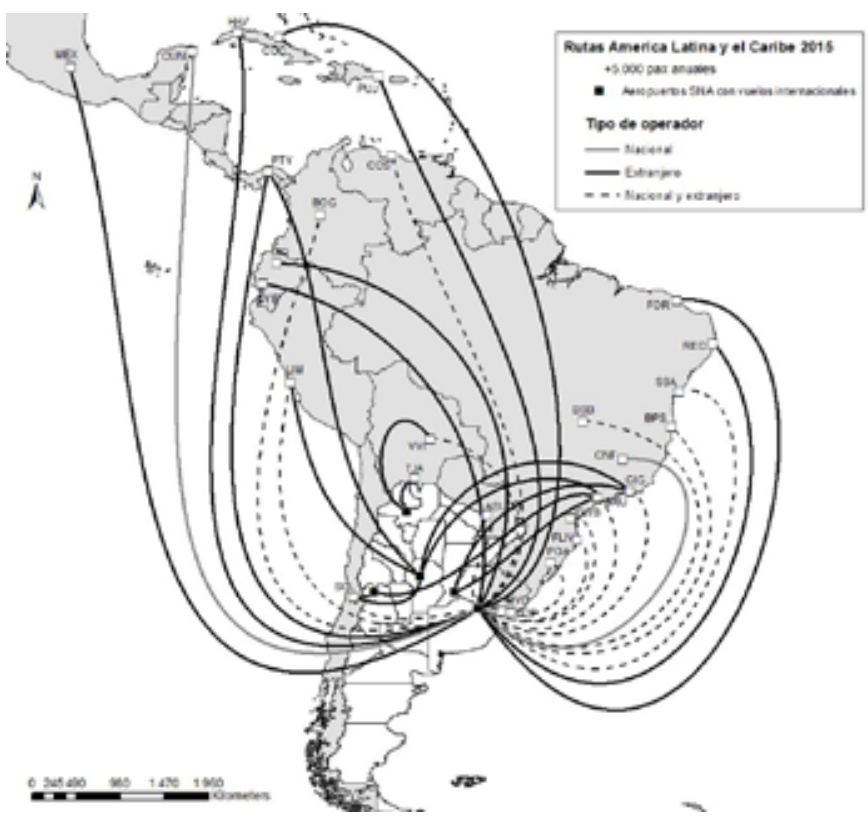

Figura 3. Red de vuelos internacionales desde/hacia América Latina y el Caribe (2015)

y Río de Janeiro (Gol). Rosario incorporó vuelos directos a Sao Paulo (LATAM/Gol) y Río de Janeiro (Gol). Salta constituyó su red en relación al flujo Argentina-Bolivia, mientras que Mendoza solo operó la ruta hacia Santiago de Chile (LATAM). Durante esos años, los principales cambios estuvieron asociados a una reconfiguración de la demanda entre Argentina y Brasil por la implementación de enlaces directos entre Buenos Aires y los principales destinos turísticos del país vecino. Mientras que en los aeropuertos porteńos se incorporaron ciudades como destino final, en los demás aeropuertos -salvo en el caso de Salta- predominó la oferta de enlaces hacia los hubs. Esta tendencia se reforzó y aceleró luego de la flexibilización del marco regulatorio, a partir de 2016.

Al comparar la red de vuelos de 2015 (Figura 3) con la de 2017 (Figura 4) se comprueba una densificación de las conexiones internacionales regionales. Los rasgos más distintivos de dicho proceso fueron: por un lado, la incorporación de nuevos aeropuertos argentinos con vuelos directos 


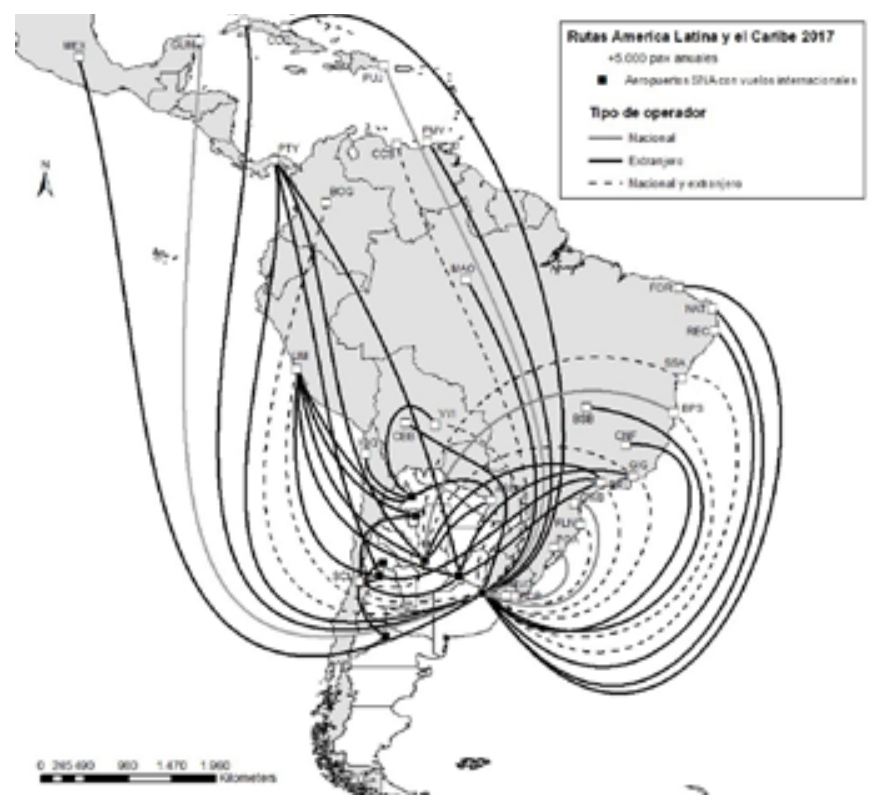

Fuente: Elaboración propia en base a datos de la ANAC.

Figura 4. Red de vuelos internacionales desde/hacia América Latina y el Caribe (2017)

hacia el exterior -Neuquén, Tucumán y San Juan- y, por otro, la proliferación de conexiones aéreas por fuera de la RMBA hacia los principales hubs de América Latina y el Caribe. Dicha descentralización de los vuelos regionales estuvo asociada a la flexibilización de los acuerdos bilaterales a partir de la cual se les otorgó a empresas extranjeras nuevas rutas y/o frecuencias desde/hacia Argentina. El hub de LATAM en Santiago de Chile sumó como destinos en el país las ciudades de Rosario, Neuquén, Tucumán y San Juan. Lima incorporó a Mendoza, Rosario, Salta y Tucumán. Panamá incluyó a Mendoza y Rosario. El caso de Córdoba fue diferente al de las demás ciudades argentinas ya que agregó vuelos directos hacia ciudades turísticas brasileras y operadores nacionales.

Como balance de todo el período, se observó un incremento de las conexiones aéreas hacia el exterior liderado fundamentalmente por las empresas extranjeras. En este escenario, la RMBA agregó nuevos destinos puntuales pero sobre todo se observó la consolidación de ciertos operadores sobre rutas específicas. Ejemplos de estos son la operación exclusiva de empresas extranjeras desde Buenos Aires hacia ciudades brasileras como: Brasilia, 


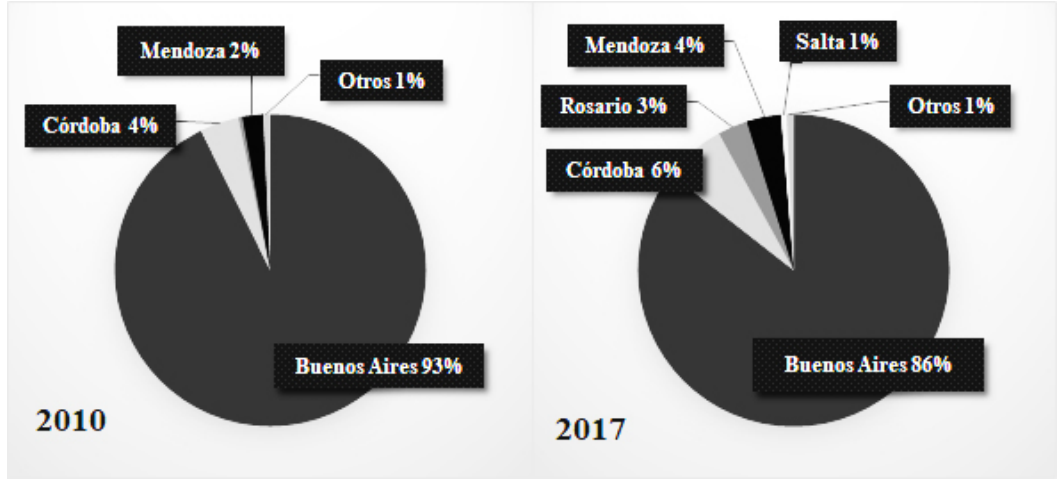

Fuente: Elaboración propia en base a datos del ORSNA.

Figura 5: Concentración de pasajeros internacionales por aeropuerto.

Belo Horizonte, Manaos, Natal. A la vez que la consolidación de las nacionales en Porto Alegre, Curitiba y Punta del Este (Uruguay).

El proceso de descentralización de vuelos repercutió considerablemente sobre los aeropuertos porteños -Aeroparque y Ezeiza- tanto en materia de conexiones aéreas donde tuvieron un crecimiento más lento que el resto de los otros nodos del país, como en la pérdida de peso relativo en el volumen total de pasajeros internacionales que circularon por Argentina. En 20I0, Buenos Aires acumulaba el 93\% de la demanda internacional de pasajeros mientras que Córdoba (4\%) y Mendoza (2\%) se ubicaban como las principales terminales del interior, el resto de los aeropuertos sumaba el I\%. En 20I7, Buenos Aires disminuyó su participación en el total sumando el $86 \%$. En paralelo, Córdoba (6\%) Mendoza (4\%), Rosario (3\%) y Salta (I\%), entre otros, incrementaron su cuota de mercado (Figura 5).

Evitando la conexión al exterior vía Buenos Aires, las ciudades antes mencionadas mejoraron sus niveles de conectividad y accesibilidad aéreas. Sin embargo, en paralelo, se produjo una extranjerización del mercado aerocomercial ya que esos nuevos enlaces fueron ofrecidos por compañías extranjeras redirigiendo el tráfico internacional hacia otros hubs de América Latina: San Pablo (LATAM/GOL), Santiago de Chile (LATAM), Lima (LATAM) y Panamá (Copa Airlines). 
Como se observa en la Figura 6, en todos los casos salvo en Buenos Aires, prácticamente la totalidad del mercado de cada ciudad fue dominado por empresas extranjeras. LATAM, Copa Airlines, Gol y Sky Airline se destacaron como las principales empresas operadoras desde otras regiones del país. La empresa chilena concentró más del 55\% del total de la demanda en todos los casos. Sólo en Buenos Aires, base operativa y centro de conexiones del Grupo Aerolíneas, la empresa nacional lideró el mercado con el $28 \%$ de los pasajeros transportados.

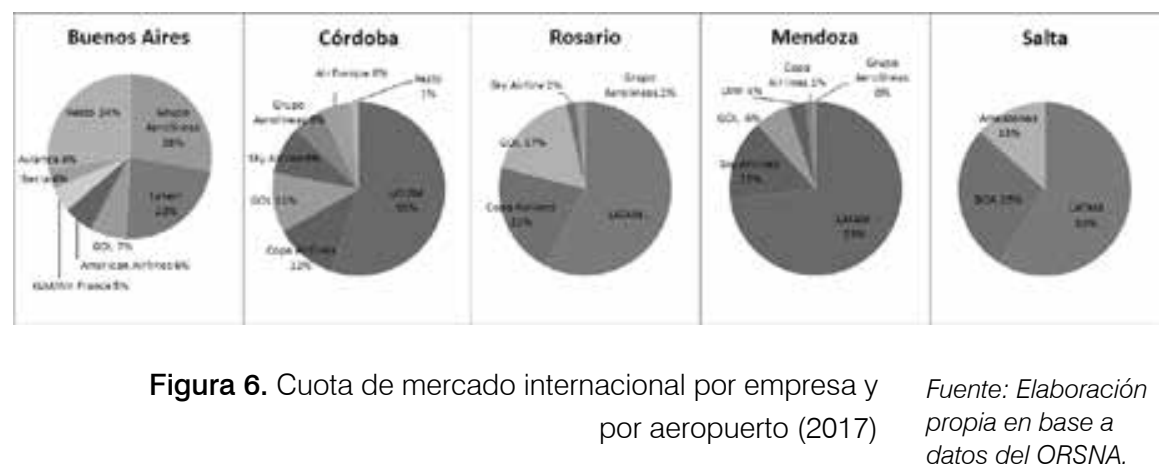

\section{Impacto económico del turismo internacional por vía aérea}

La demanda aérea y los flujos turísticos

Aunque los pasajeros internacionales tuvieron un desempeño positivo entre 20I0 y 2017, los flujos del turismo ${ }^{3}$ emisivo y receptivo por vía aérea mostraron una tendencia contrapuesta (Figura 7). Por un lado, los turistas residentes que viajaron al exterior crecieron significativamente, sobre todo luego de 20I4. En 20IO, hubo 2.206,7 millones de turistas emisivos, mientras que en 2017 fueron 4.516,I millones, creciendo un 105\%. Por otro, los turistas no residentes que llegaron a Argentina se redujeron un $-9 \%$, pasando de 2.8I8,5 millones (2010) a 2.567,0 millones (2017).

3 Turista internacional es todo visitante internacional que pasa por lo menos una noche en el lugar visitado. Recuperado de: https://www.indec.gob.ar/ftp/cuadros/economia/eti_ aspectos_metodologicos_2018.pdf 
Como resultado, la importancia del turismo receptivo se redujo a partir 2012 y la brecha entre ambos flujos creció de forma sostenida. En 20IO, los viajeros no residentes representaban un $56 \%$ del flujo turístico total mientras que en 2017 solo un $36 \%$. En cambio, el turismo emisivo evolucionó positivamente pasando del $44 \%$ en 2010 al $64 \%$ del turismo internacional por vía aérea en 2017.

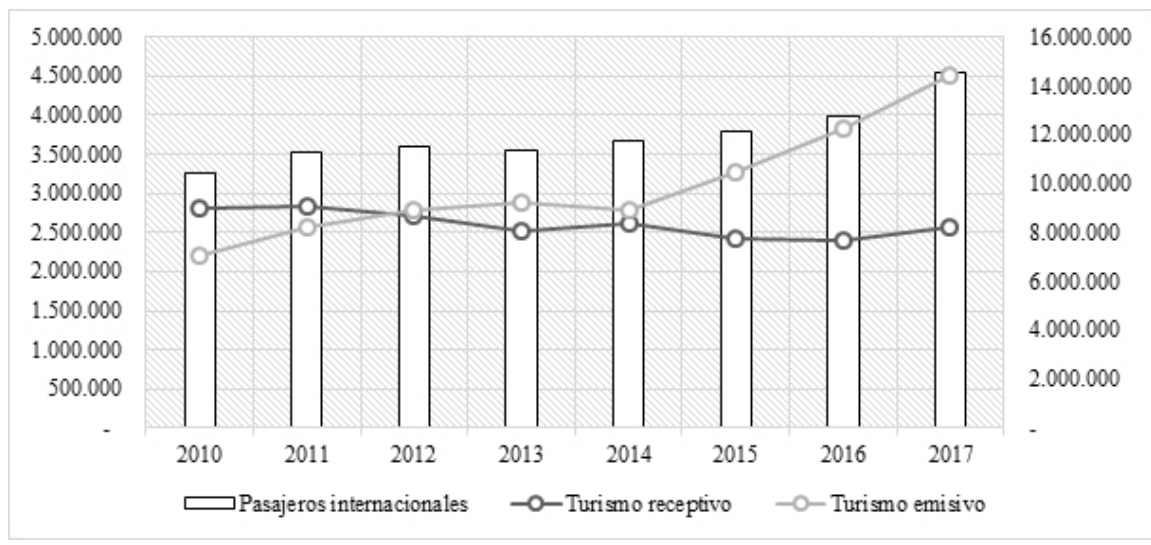

Fuente: Elaboración propia en base a datos del Instituto Nacional de Estadística y Censos (INDEC)
Figura 7: Pasajeros internacionales, turismo receptivo y emisivo por vía aérea (2010-2017)

Adicionalmente, los cambios en la red aerocomercial generaron transformaciones en la distribución geográfica de los flujos. Como muestra la Figura 8, Buenos Aires en 2010 acumulaba el 94\% del turismo receptivo y el $86 \%$ del turismo emisivo por vía aérea de todo el país. En 20I7, su participación había bajado al 92\% y el $83 \%$ respectivamente. En particular, los aeropuertos por fuera de la RMBA duplicaron la cantidad de tráfico internacional pasando de 476.4I2 (20IO) turistas aéreos a 99I.908 (20I7), es decir, que pasaron a representar el I4\% del total del flujo, mientras que antes solo contaban con el $9 \%$. A su vez, este crecimiento estuvo marcado por una mayor participación de estos aeropuertos en el flujo emisivo ( $17 \%)$ que en el receptivo $(8 \%)$. 

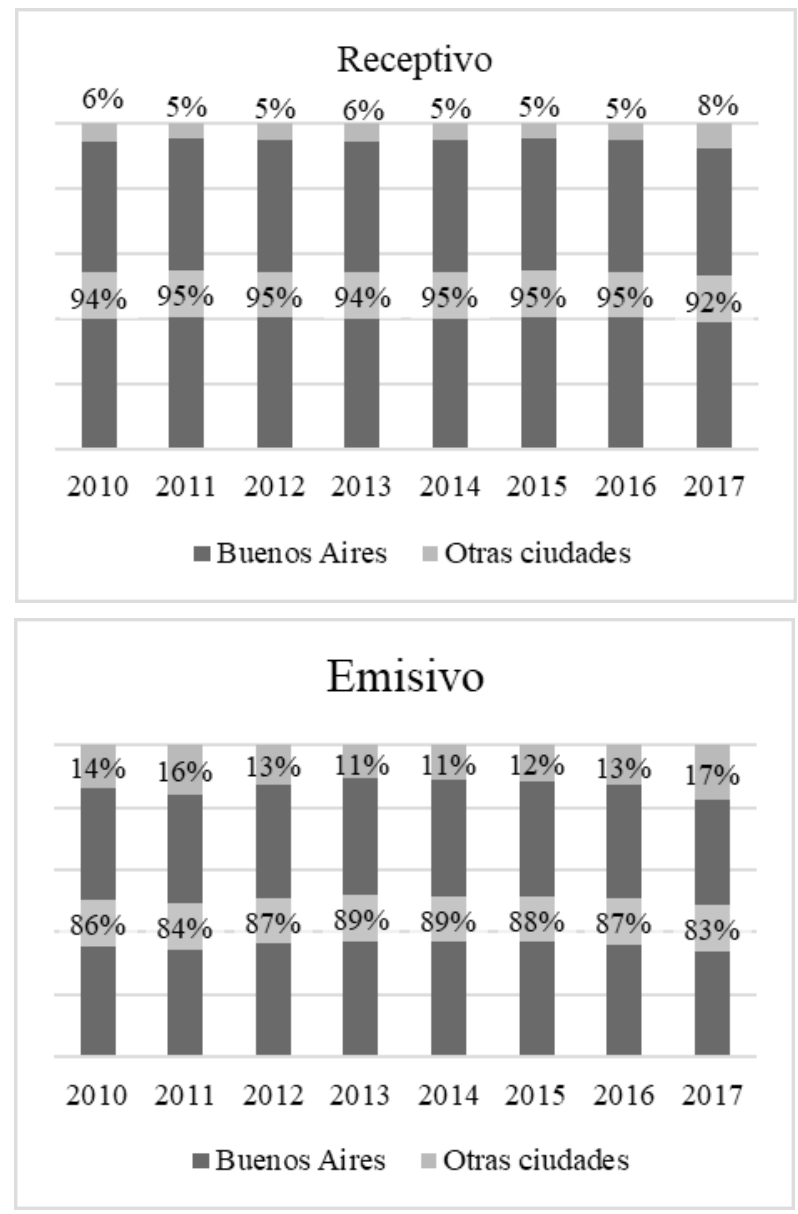

Fuente: Elaboración propia en base a datos del INDEC.

Figura 8: Participación del turismo receptivo y emisivo por vía aérea según destino (2010-2017)

Cuando se mira la composición del flujo turístico de 2017 queda claro que todas las terminales aéreas fueron utilizadas mayormente por residentes para viajar al exterior (Figura 9). Salvo Buenos Aires, los principales aeropuertos manifestaron un desbalance del saldo turístico importante a favor del turismo emisivo donde los casos más extremos fueron Rosario (89\%), Córdoba $(84 \%)$ y Salta (84\%). En ningún caso el turismo receptivo superó el 40\%, sin embargo, los aeropuertos de Buenos Aires fueron los que mayor visitantes atrajeron. En números absolutos, 2,3 millones de extranjeros ingresaron a través de Buenos Aires, es decir, el 92\% del total país. 


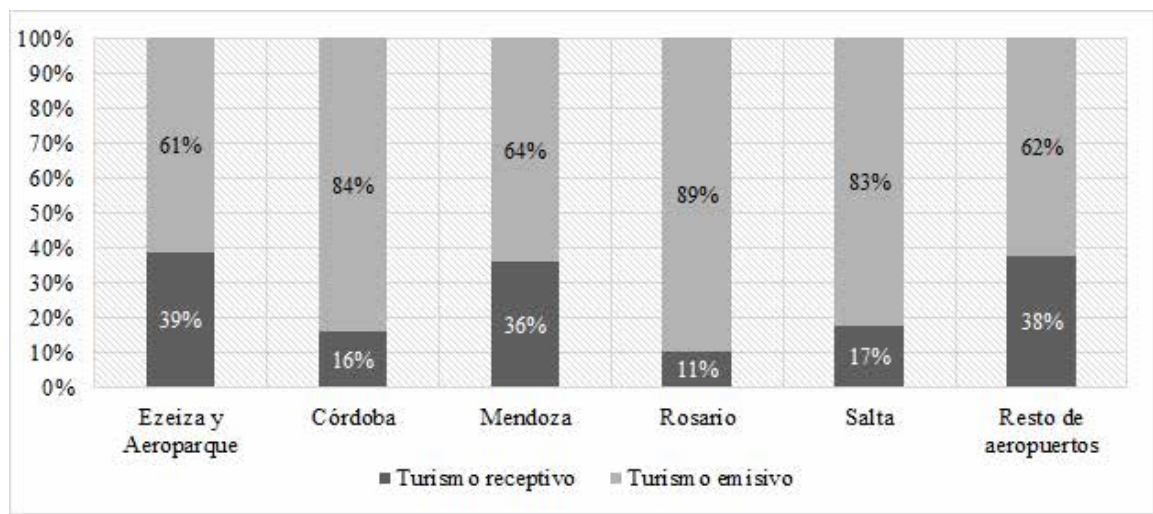

Fuente: Elaboración propia en base a datos del INDEC.

Figura 9: Participación del turismo emisivo y receptivo en el flujo turístico total de cada aeropuerto (2017)

La suma de nuevos enlaces directos y el aumento de las frecuencias en ciudades como Córdoba, Rosario, Mendoza, Salta, Tucumán, Neuquén, entre otras, redundó en una mejora de la conectividad y accesibilidad aéreas que benefició sobre todo al turismo emisivo.

El saldo negativo del turismo receptivo entre 2010 y 2017 mostró que la contracción de los no residentes que visitaron Argentina estuvo mayormente vinculada con una caída de la demanda vía Buenos Aires del -II\% (Figura Io). En contraste, el turismo receptivo desde los demás aeropuertos creció un $2 \mathrm{I} \%$.

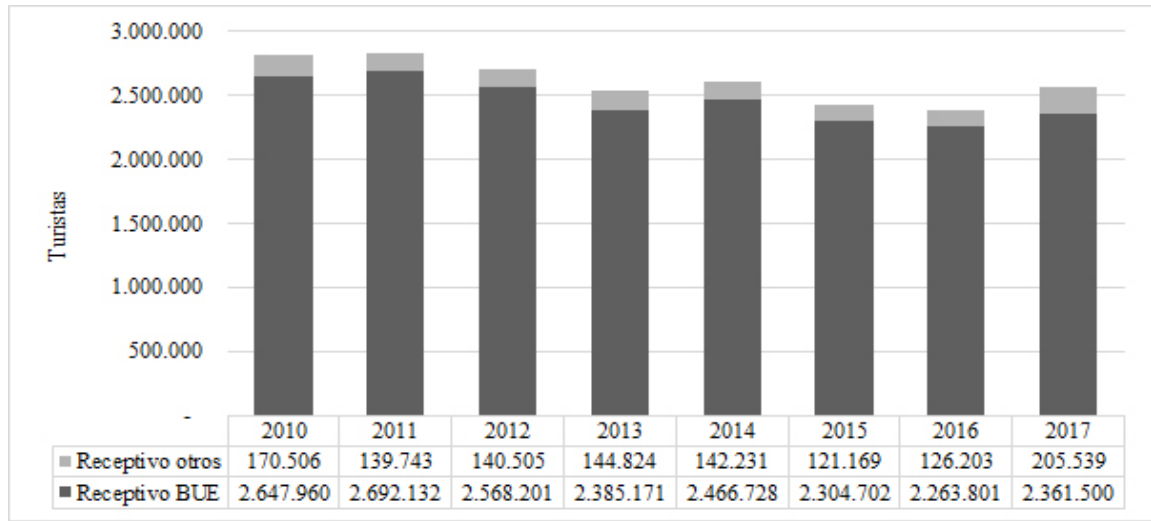

Fuente: Elaboración propia en base a datos del INDEC.
Figura 10. Distribución del turismo receptivo por vía aérea según ciudades (2010-2017) 
En cambio, la cantidad de argentinos que viajaron al exterior se incrementó en todos los aeropuertos. En la RMBA creció un 96\% y en los demás nodos aeroportuarios un $157 \%$ (Figura II). No obstante, es interesante destacar que de los 2,3 millones de residentes adicionales que viajaron al exterior entre 2010 y 2017 , el $54 \%$ se sumó recién a partir de 20I5. Es decir, entre 2015 y 2017 se sumaron I,250 millones de turistas emisivos y de ese total el 33\% -409.547-correspondió a viajeros que salieron desde los aeropuertos del interior del país.

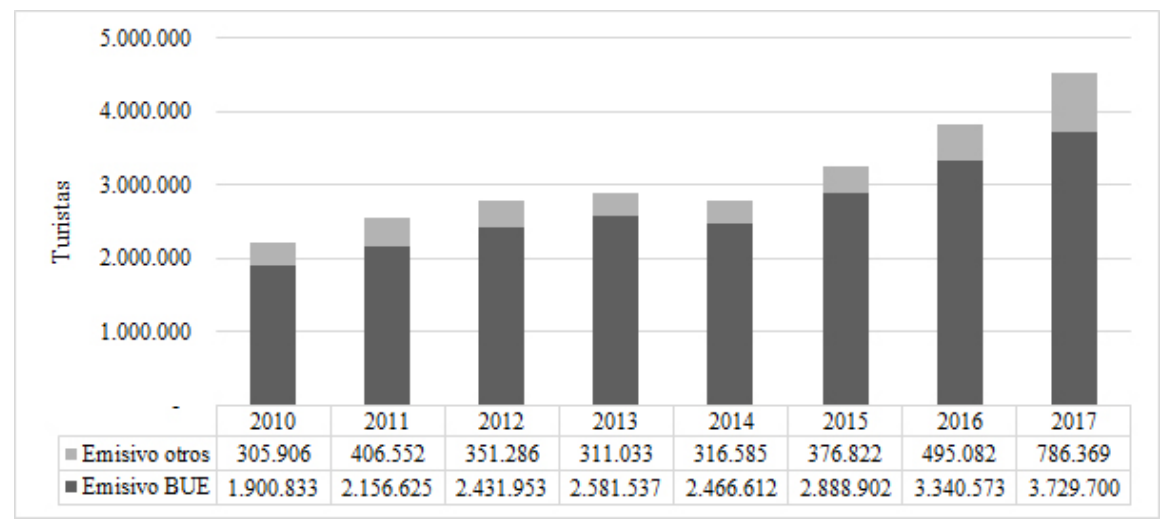

Figura 11. Distribución del turismo receptivo por vía aérea según ciudades (2010-2017)

Fuente: Elaboración propia en base a datos del INDEC.

La contribución neta del turismo por vía aérea

Los aeropuertos que cuentan con datos históricos (20IO-20I7) sobre el gasto realizado por el turismo internacional son el Aeroparque Jorge Newbery, el Aeropuerto Internacional de Ezeiza y el Aeropuerto Internacional de Córdoba. Estas terminales representan actualmente más del 90\% del total de pasajeros internacionales que llega y parte de Argentina por lo que sus cifras son más que representativas para tener una aproximación al impacto catalítico del turismo internacional por vía aérea.

Como se apuntó, desde 2012 el saldo de turistas llegados y salidos a través de los aeropuertos porteños ha mostrado resultados negativos, excepto en el año 20I4. A su vez, esta tendencia se agravó en los últimos dos años. En sintonía, la Figura I2 muestra cómo evolucionó entre 2010 y 2017 el saldo del económico en materia turística asociado a esos aeropuertos. 


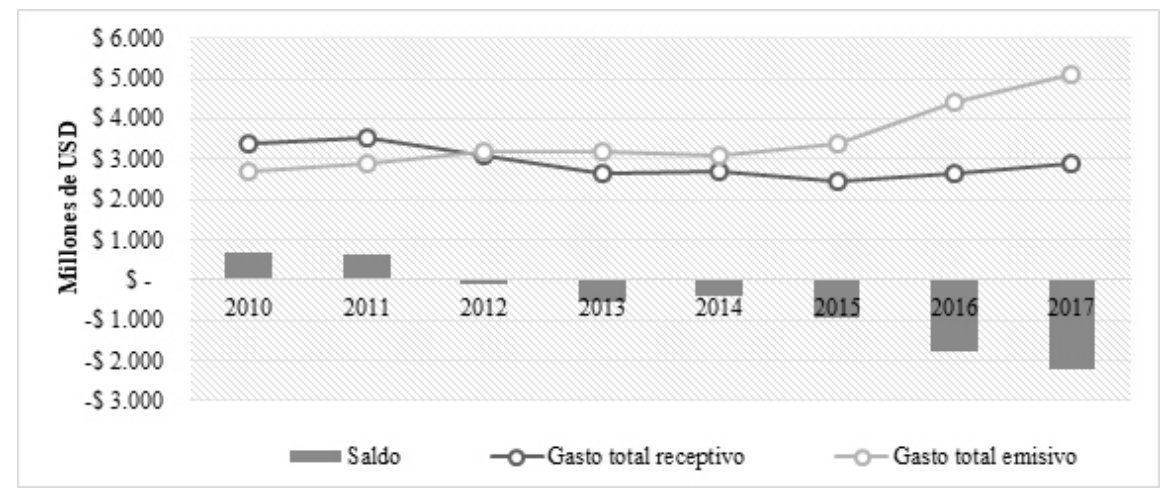

Fuente: Elaboración propia en base a datos del INDEC.
Figura 12: Impacto económico de los viajeros internacionales por vía aérea - Aeropuerto Internacional de Ezeiza y Aeroparque Jorge Newbery (2010-2017)

Según los datos del INDEC, el volumen de gasto de los argentinos en el exterior -impacto negativo- creció un 91\%, mientras que el gasto de los turistas extranjeros en Argentina -impacto positivo- decreció un I4\%. En 20Io, el turismo emisivo contabilizó un gasto total de \$2.673,5 millones de dólares, mientras que en 2017 superó los \$ 5.097,4 millones de dólares. En cambio, el turismo receptivo generó un ingreso de \$3.369,6 millones de dólares en 2010 y de \$2.902,0 millones de dólares en 2017 .

En síntesis, la contribución neta del turismo por vía aérea de Buenos Aires mostró un saldo negativo creciente desde el año 20I2. Entre 2012 y 2017, la balanza comercial turística de los aeropuertos porteños pasó de $-\$ 89,6$ millones de dólares a $-\$ 2.195,4$ millones de dólares, con un crecimiento del $2.449 \%$.

En la ciudad de Córdoba sucedió lo mismo (Figura I3): entre 2010 y 2017 tanto el déficit turístico como el saldo económico negativo se incrementaron. Los pasajeros internacionales de Córdoba crecieron un iı 6\%. A su vez, los viajeros no residentes aumentaron un $43 \%$, mientras que los residentes que viajaron al exterior crecieron un $160 \%$. Como resultado, el saldo de flujos turísticos -que ya era negativo en 20Io- se acrecentó un $221 \%$ en 20I7, y la contribución neta del turismo internacional profundizó su saldo negativo aumentando un 210\% en esos años. 


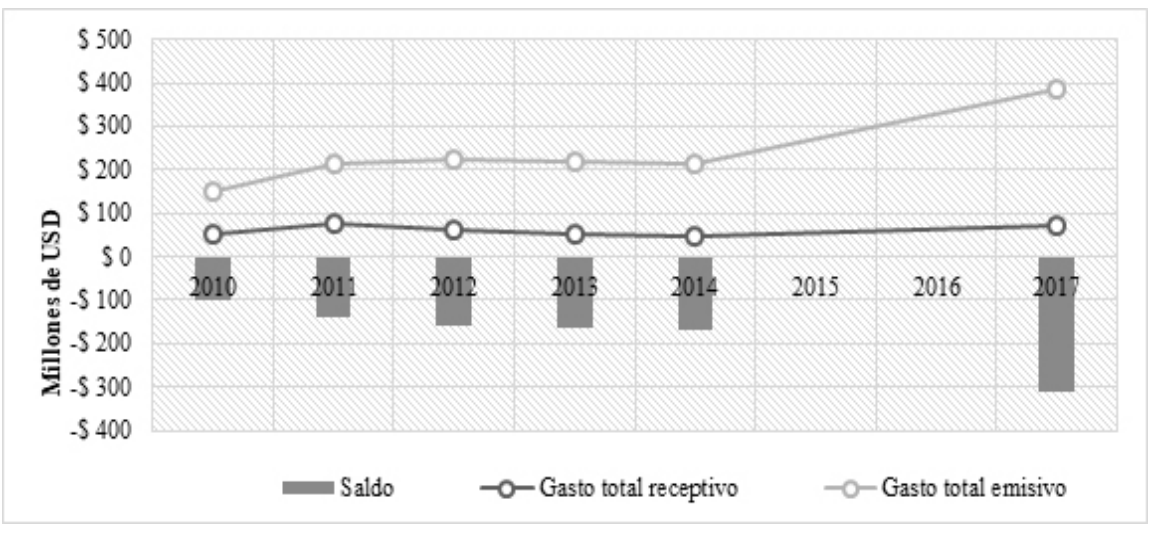

Figura 13: Saldo turístico e impacto económico de los viajeros internacionales - Aeropuerto Internacional Córdoba (2010-2017)

Fuente: Elaboración propia en base a datos del INDEC.

En resumen y teniendo en cuenta los tres aeropuertos, la contribución neta del turismo internacional entre 2010 y 2017 mostró un crecimiento sostenido del saldo negativo, alcanzando cifras récord en 2017 con un déficit de $-\$ 2.507,7$ millones de dólares (Figura I4). A este cálculo debería agregarse el saldo de impactos de los demás aeropuertos que fueron ganando mercado internacional como Rosario, Salta, Mendoza, entre otros. Aunque no hay información disponible sobre el gasto de residentes y no residentes para esos aeropuertos se estima que por la composición del flujo en cada caso -preeminencia del turismo emisivola contribución neta sea negativa. En consecuencia, el saldo total para el país sería aún más deficitario. Como balance del período, el turismo internacional por vía aérea pasó de ser una actividad con aportes monetarios positivos para la economía nacional, a representar una vía importante de fuga de divisas hacia otros países. 


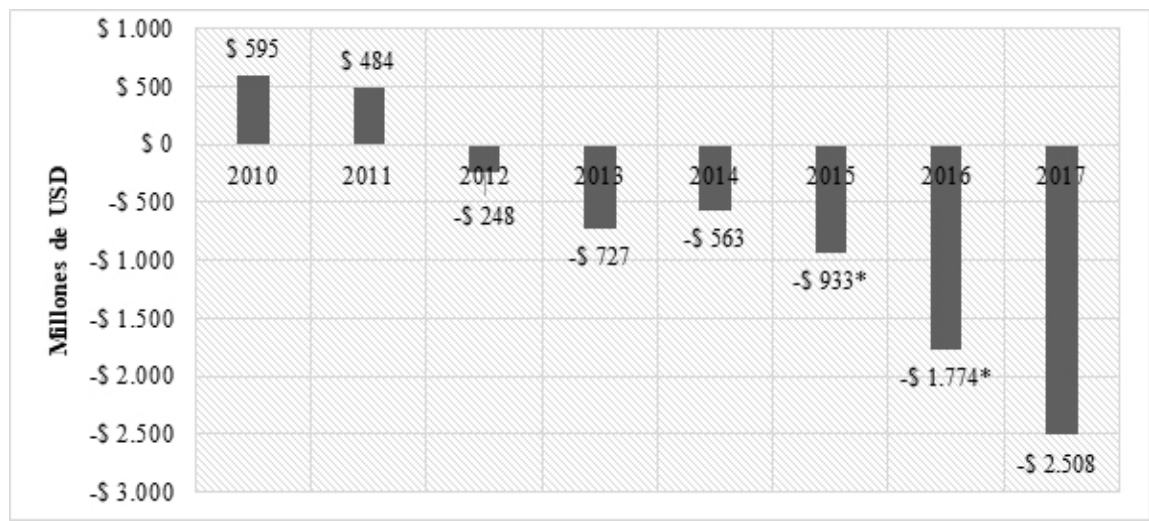

Fuente: Elaboración propia en base a datos del INDEC
Figura 14: Contribución neta del turismo internacional por vía aérea (2010 vs 2017)

*No hay datos disponibles para el Aeropuerto Internacional de Córdoba.

Desequilibrios en la cuenta corriente de los servicios de transporte aéreo

Por último, el desequilibrio económico de la balanza de viajes por turismo aéreo internacional se vio reforzado por las transacciones realizadas por los turistas residentes y no residentes en la compra de servicios de transporte aéreo. Como se mencionó, el incremento de la conectividad internacional en los últimos años estuvo liderado por líneas aéreas extranjeras. Dicha situación, redundó en una mayor extranjerización del mercado favoreciendo la remesa de utilidades a las casas centrales de las compañías latinoamericanas en el exterior. Esta hipótesis se constata al analizar cuenta corriente de la balanza de pagos argentina.

La cuenta corriente de servicios de transporte registra todos los ingresos (crédito) y egresos (débito) derivados de servicios prestados entre residentes y no residentes. La cuenta corriente de servicios de transporte aéreo contabiliza tanto el valor de los pasajes vendidos por las compañías aéreas argentinas a turistas no residentes (crédito) como el valor de los pasajes vendidos por las compañías aéreas extranjeras a viajeros residentes (débito). Al saldo de pasajes se le suma también el saldo del flete aéreo y otros servicios como control de tráfico aéreo, limpieza de equipo de transporte, tasas por el uso del aeropuerto, rampas y comisiones de agentes (INDEC, 20I8:15). 
La Figura I5 muestra la evolución de los ingresos, egresos y el consecuente saldo de los servicios aéreos. El déficit de la cuenta corriente se duplicó durante el período 20I0-2016 pasando de -944,9 millones de dólares (2010) a -I.896,5 millones de dólares (20I6). Pero, además, dentro del saldo general, la venta de pasajes fue el ítem con mayor déficit de la cuenta corriente de servicios de transporte aéreo (Figura I6). Esto refleja la transferencia de utilidades hacia el exterior a través de las compañías extranjeras que operan servicios internacionales en Argentina. En 20I6, el ingreso de divisas por venta de pasajes a no residentes a través de compañías nacionales fue de \$462,55 millones de dólares. En cambio, el egreso de divisas por venta de tickets a residentes vía empresas extranjeras fue de \$2.599,65 millones de dólares.

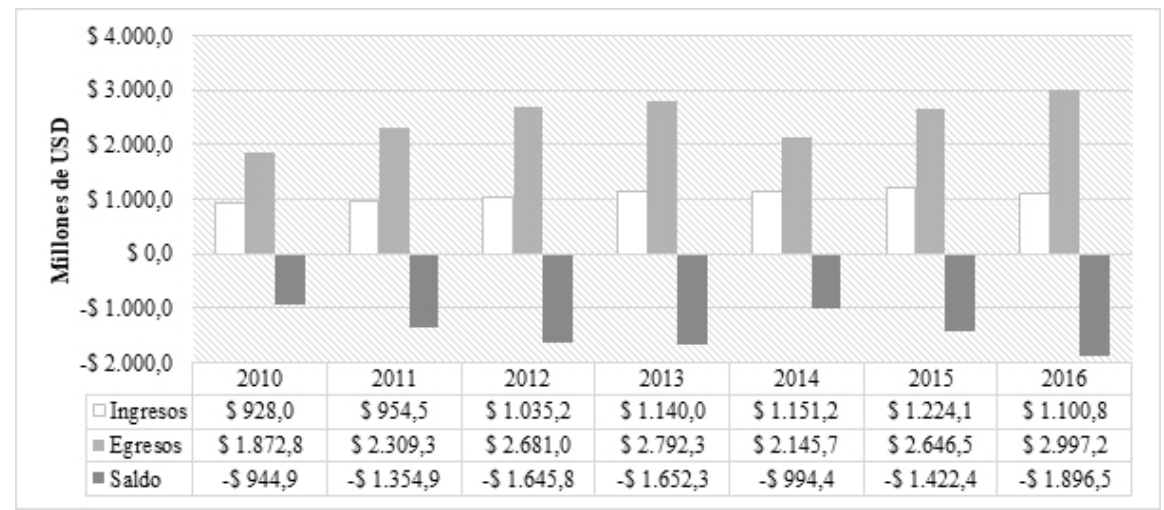

Figura 15: Ingresos, egresos y saldo de los servicios de Fuente: Elaboración transporte aéreo (2010-2016) 


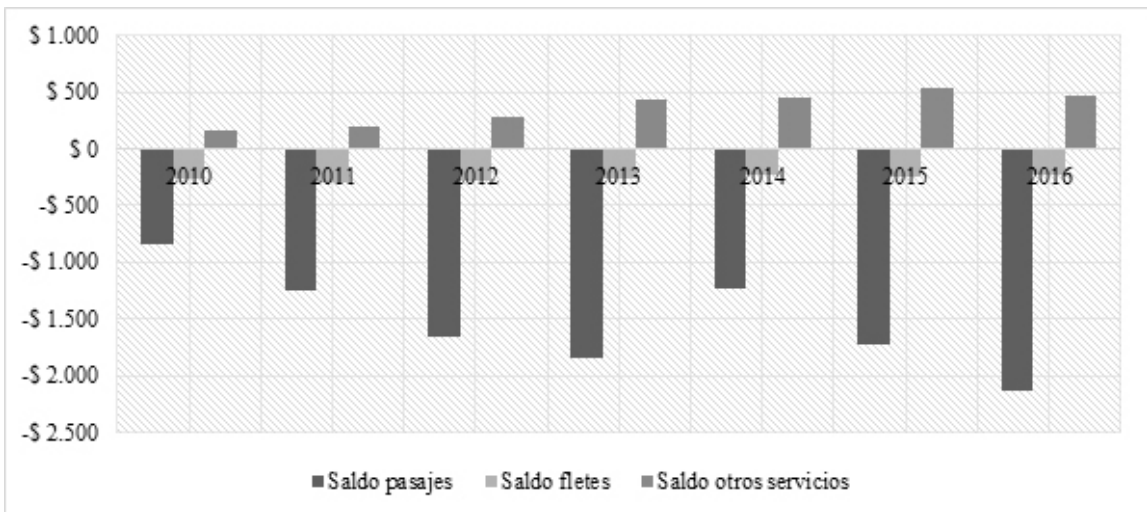

Fuente: Elaboración propia en base a datos del INDEC.

Figura 16: Saldo en pasajes, flete y otros servicios - Cuenta corriente (2010-2016)

\section{Reflexiones finales}

Abordar la relación entre transporte aéreo y turismo implica poner en juego variadas dimensiones de análisis. Este trabajo se propuso cuantificar el efecto económico generado por los flujos turísticos en el territorio nacional desde una perspectiva de saldo de impactos (receptivo-emisivo). No obstante, también buscó poner en discusión los alcances tanto en materia de oferta de servicios como de beneficios económicos-territoriales que generan cierto tipo de (des)regulaciones dentro del sector que tradicionalmente se entienden como promotoras de la actividad turística, por ejemplo, la liberalización del mercado aerocomercial. En este sentido, el rol del Estado es fundamental tanto en la configuración de las redes de líneas aéreas como en la determinación del acceso a los centros de distribución de vuelos (Bowen, 2000), lo cual tiene consecuencias sobre el impacto económico-territorial generado por el transporte aéreo.

Las modificaciones del marco regulatorio en 2016 buscaron ganar un mayor grado de liberalización y apertura del mercado aéreo como herramienta de desarrollo económico a través de: la renegociación de acuerdos bilaterales, la habilitación de nuevas rutas y frecuencias para las compañías extranjeras, y la descentralización de la operación de los vuelos internacionales. Estos procesos incrementaron la cantidad de servicios aéreos 
ofrecidos desde/hacia Argentina, promovieron la participación de otros aeropuertos del país en el flujo total de turistas internacionales y produjeron una mejora en la conectividad y accesibilidad aéreas de ciudades de Córdoba, Rosario, Mendoza, Salta, entre otras.

No obstante, la contribución neta del turismo internacional por vía aérea, empeoró paulatinamente entre 20I0 y 20I7. El flujo turístico que a principios del período mostraba una prevalencia del turismo receptivo sobre el emisivo y un superávit de la balanza comercial turística asociada al gasto de los residentes y no residentes cambió rotundamente a partir de 2012 acentuando la prevalencia del turismo emisivo y la transferencia de divisas hacia el exterior. Este escenario se fue agravando progresivamente y se profundizó entre 2016 y 2017 cuando se alcanzó un déficit record: $-\$ 2.507,7$ millones de dólares.

Adicionalmente, la creciente extranjerización del mercado aéreo internacional profundizó el saldo negativo asociado a la venta de tickets aéreos entre residentes y no residentes transfiriendo utilidades hacia el exterior a través de las empresas extranjeras con base en otros países. Este déficit de la cuenta corriente de servicios de transporte aéreo conjugó varios procesos: la preeminencia del turismo emisivo en el flujo total turístico, el liderazgo de las empresas extranjeras en la incorporación de nuevas conexiones aéreas y la descentralización geográfica de los vuelos. Particularmente, la operación de vuelos directos desde las ciudades del interior hacia los centros de distribución de vuelos de las grandes empresas multilatinas desincentivó la operación vía Buenos Aires y repercutió en la caída en la cuota de mercado del Grupo Aerolíneas -empresa cuyo hub doméstico e internacional tiene sede en dicha ciudad-. Este escenario obstaculizó el impacto económico genuino vinculado a la oferta aérea brindada por líneas aéreas con base en el país y estrategias operativas basadas en hubs locales.

A modo de cierre, el análisis precedente invita a rediscutir los alcances deseados y no deseados de la regulación vigente y a pensar nuevas estrategias para promover la generación de impactos económicos genuinos vinculados al turismo receptivo y a la cualidad de la oferta aerocomercial. Dichas estrategias podrían contemplar la necesidad de estimular la llegada de extranjeros a través de los nodos provinciales para compensar el flujo emisivo, incentivar la oferta de servicios aéreos nacionales y/o fortalecer el hub Buenos Aires, ya que es la principal vía de entrada de turistas no residentes y base de operaciones de líneas aéreas locales. 


\section{Referencias bibliográficas}

ACI, ATAG, CANSO, IATA, ICAO e ICCAIA. (2017). Aviation benefits 2017.

[En línea] Recuperado de: https://www.iata.org/policy/Documents/aviationbenefits-\%2oweb.pdf

\section{Airports Council International Europe, ACI-Europe e Intervistas. (2015).} Economic Impact of European Airports. A critical catalyst to Economic Growth. Intervistas.

Arias, F., y Lipovich, G. (2017). Los límites del crecimiento aerocomercial y aeroportuario en la Región Metropolitana de Buenos Aires, en Müller, A. Transporte urbano e interurbano en la Argentina. Aportes desde la investigación, Buenos Aires: EUDEBA.

Bowen, J. (2000). Airline hubs in Southeast Asia: national economic development and nodal accessibility, Journal of Transport Geography, N ${ }^{\circ}$, pp. 25-4I, Reino Unido: Elsevier.

(20I4). The Economic Geography of Air Transport, en Goetz, A. y Budd, L. (eds.) The Geographies of air transport, Dorchester, Reino Unido: Ashgate.

Dobruszkes, F. (2014). Geographies of European Air Transport, en Goetz, A. y Budd, L. (eds.) The Geographies of air transport, Dorchester, Reino Unido: Ashgate.

Dobruszkes, F., Mondou, V. y Ghedira, A. (2016). Assessing the impacts of aviation liberalisation on tourism: Some methodological considerations derived from the Moroccan and Tunisian cases, Journal of Transport Geography, Vol. 50, pp. II5-I27, Reino Unido: Elsevier.

Duval, D.T. (2013). Critical Issues in Air Transport and Tourism, Tourism Geographies: An International Journal of Tourism Space, Place and Environment, Vol. I5, No. 3, (pp. 494-510) London: Routledge.

Duval, D. T. (2018). "The Impact of Government Policy and Regulation», en Graham, A. y Dobruszkes, F. (eds.) Air Transport-A Tourism Perspective, Elsevier [eBook].

Forsyth, P. J. (2008). Tourism and aviation policy: Exploring the links, en Graham, A.; Papatheodorou, A. y Forsyth, P. (eds.), Aviation and Tourism: Implications for Leisure Travel, Aldershot England: Ashgate Publishing Limited.

Gámir, A. y Ramos, D. (2002). Transporte aéreo y territorio. Barcelona: Ariel.

Goetz, A. (2002). Deregulation, competition, and antitrust implications in the US airline industry, Journal of Transport Geography, Vol ıo, N ${ }^{\circ}$, pp. I-I9, Reino Unido: Elsevier. 
Goetz, A. y Vowles, T. (2009). The good, the bad, and the ugly: 30 years of US airline deregulation, Journal of Transport Geography, N${ }^{\circ}$, pp. 25I-263, Reino Unido: Elsevier.

Graham, B. (I995). Geography and Air Transport. Chichester, Reino Unido: Editorial Wiley.

International Air Transport Association, IATA (2017). The importance of air transport in Argentina [En línea] Recuperado de: https://www.iata.org/ policy/Documents/benefits-of-aviation-argentina-20I7.pdf

Instituto Nacional de Estadística y Censos, INDEC (2018). Balanza de pagos, posición de inversión internacional y deuda externa. Recuperado de: https:// www.indec.gob.ar/ftp/cuadros/economia/bdp_pii_de_metodologia_23.pdf

Lipovich, G. (2002). ¿Concentración en los buenos aires del Mercosur? El papel de Buenos Aires en el transporte aéreo dentro de la región, IX Jornadas Cuyanas de Geografía. Mendoza.

- (2010). Los aeropuertos de Buenos Aires y su relación con el espacio metropolitano. La inserción del subsistema aeroportuario Aeroparque-Ezeiza dentro de la lógica del mercado aerocomercial y de la estructuración urbana. Buenos Aires: Facultad de Filosofía y Letras-UBA.

(2014). Geographies of Latin American Air Transport, en Goetz, A. y Budd, L. (eds.) The Geographies of air transport, Dorchester, Reino Unido: Ashgate.

Organización Mundial del Turismo (2018). UNWTO Tourism Highlights. 2017 Edition. Disponible online: https://www.e-unwto.org/doi/ pdf/IO.I8III/9789284419029

ORSNA (2015). Informe de Impacto Económico-Territorial del Aeropuerto Internacional de Córdoba, Departamento de Análisis Económico-Territorial y Estadística. Buenos Aires: ORSNA. http://www.orsna.gov.ar/informes/IETCOR\%202013.pdf

Redondi, R.; Malighetti, P.y Paleari, S. (20II). «Hub competition and travel times in the world-wide airport network", Journal of Transport Geography, $\mathrm{N}^{\circ}$ 19, pp. 1260-I27I, Reino Unido: Elsevier.

UNWTO (2012). Global Report on Aviation: Responding to the Needs of New Tourism Markets and Destinations. UNWTO, Madrid.

Wallingre, N. (20I0). Responsabilidad del transporte aéreo en la dinámica territorial del turismo argentino. El Aporte pendiente, Revista Transporte y Territorio, $\mathrm{N}^{\circ}$ 2, Buenos Aires: Universidad de Buenos Aires. 